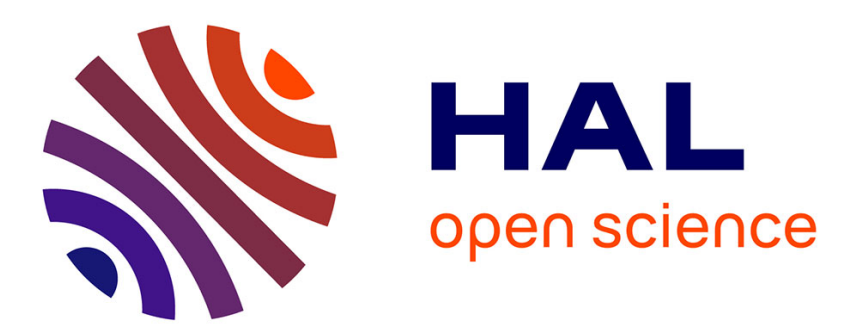

\title{
Faut-il remettre les pendules de la subordination temporelle à l'heure? Description de deux fonctionnements de quand et avant que/de
}

Christophe Benzitoun

\section{- To cite this version:}

Christophe Benzitoun. Faut-il remettre les pendules de la subordination temporelle à l'heure? Description de deux fonctionnements de quand et avant que/de. Cahiers Chronos, 2013, 26, pp.419-435. hal-00931848

\author{
HAL Id: hal-00931848 \\ https://hal.science/hal-00931848
}

Submitted on 15 Jan 2014

HAL is a multi-disciplinary open access archive for the deposit and dissemination of scientific research documents, whether they are published or not. The documents may come from teaching and research institutions in France or abroad, or from public or private research centers.
L'archive ouverte pluridisciplinaire HAL, est destinée au dépôt et à la diffusion de documents scientifiques de niveau recherche, publiés ou non, émanant des établissements d'enseignement et de recherche français ou étrangers, des laboratoires publics ou privés. 


\title{
Faut-il remettre les pendules de la subordination temporelleà l'heure? Description de deux fonctionnements de quand et avant que/de
}

\author{
Christophe BENZITOUN \\ Université de Nancy 2 \& ATILF CNRS
}

\section{Introduction}

Cela fait bien longtemps que les linguistes travaillant sur le français ont remarqué un usage singulier de quand n'exprimant pas une simple localisation temporelle. Cet emploi a reçu originellement le nom de «quand de péripétie ( Séchehaye 1926) puis «subordination inverse». L'exemple suivant en constitue une bonne illustration :

\section{(1) Pécuchet venait d'en remettre la note à Bouvard quand tout à coup le tonnerre retentit et la pluie tomba (roman)}

Dans cette configuration, la construction introduite par quand (que nous abrègerons désormais en Quand-C) ne constitue pas un repère temporel, contrairement aux exemples « canoniques » du type :

(2) Il nous a abandonnés, ma mère et moi, quand j'avais à peine un an. (roman)

Plus récemment, Le Draoulec (2003) et Delort (à paraitre ${ }_{a}$ ) se sont intéressées à un fonctionnement impliquant avant que et avant de ressemblant étrangement à (1).

(3) 61 personnes, sur les 111 salariés présents sur le site chalonnais hier, ont débrayé de $14 \mathrm{~h}$ à $15 \mathrm{~h}$, avant de reprendre ensuite normalement leur travail. (presse)

De la même façon, il s'oppose aux exemples en avant que/de fonctionnant comme un repère temporel :

Le 6 juillet, avant de me coucher, j'ai placé sur ma table du vin, du lait, de l'eau, du pain et des fraises. (roman)

Ces deux fonctionnements de quand et avant que/de ont fait l'objet de nombreuses analyses pragmatiques, sémantiques et discursives, si bien que l'on est désormais capable de caractériser précisément ces deux interprétations à travers notamment la distinction entre la présupposition et l'assertion (cf. Le Draoulec 2003 \& 2006). Mais personne, ou presque, n'a abordé de manière précise les propriétés syntaxiques de ces constructions, excepté Benzitoun (2006) (sur quand uniquement). Nous nous proposons donc de poursuivre ce premier travail en mettant en évidence les caractéristiques syntaxiques des constructions en avant que/de et quand du même type que celles des exemples ci-dessus.Nous montrerons notamment qu'il existe une congruence entre syntaxe et sémantique, pour peu que le cadre syntaxique que l'on adopte ne soit pas trop imprégné de la conception scolaire de la subordination. Nous espérons ainsi parvenir à «remettre les pendules à l'heure » entre la syntaxe et la sémantique afin d'approfondir la question de leur interaction. Néanmoins, nous nous limiterons à l'étude spécifique des deux fonctionnements exemplifiés ci-dessus. Cela nous permettra de poser les prémisses d'un travail plus ambitieux concernant l'interface entre la syntaxe et la sémantique.

Dans la première partie, nous retracerons l'histoire de la «subordination inverse » et des différents termes successifs qui ont été employés pour désigner les exemples en quand tels que (1), afin de mettre en lumière leur caractère polysémique et généralement contradictoire. Dans la deuxième partie, nous rappellerons brièvement les principales propriétés dégagées dans les études sémantiques. Dans la troisième partie, nous proposerons une analyse qui montre que l'opposition sémantique entre présupposition et assertion est doublée d'une opposition syntaxique entre dépendance et autonomie. Enfin, dans la dernière partie, nous proposerons des pistes de recherche portant sur d'autres exemples.

\section{Brève histoire de la « subordination inverse »}

Dans cette section, nous allons montrer qu'il est difficile de cerner avec précision les éléments pouvant être considérés comme appartenant à la «subordination inverse » et que la variabilité entre les auteurs est assez importante, ce qu'illustre bien la terminologie usitée ou créée : «quand de péripétie », « subordination inverse », «quand inverse », «subordination à valeur coordonnante» ou encore «quand narratif ». Le moins que l'on puisse dire, c'est que cette tournure a suscité une grande profusion terminologique. Mais, en réalité, nous verrons que ces termes, parfois à l'insu de leur auteur, ne désignent pas exactement les mêmes constructions, ou plutôt qu'ils n'ont pas nécessairement la même portée. Par un processus de dérive sémantique, un auteur reprenant à 
son compte une notion définie par un autre n'en fait pas exactement le même usage, si bien qu'au final, non seulement la notion s'obscurcit mais il devient en plus très difficile de l'employer sans équivoque.

En fait, la notion de «subordination inverse » avait fondamentalement pour utilité d'englober certaines configurations ne correspondant pas à la définition générale de la «subordination ».Mais nous verrons qu'il y a des exceptions aux règles prescrites pour la «subordination inverse », ce qui amène certains auteurs à créer encore une autre notion permettant d'englober les exceptions des exceptions, sans que l'on puisse juger de la cohérence du cadre d'ensemble.

Au départ, il semble que ce soit A. Séchehaye qui, le premier, en 1926, a remarqué une tournure étrange introduite par quand ou lorsque en français contemporain. Il en a donné la description suivante :

Il s'agit en fait d'une simultanéité ou d'un rapport réciproque à la faveur duquel se produit un renversement du mouvement des idées. Bien qu'ayant gardé la forme d'une principale suivie de sa subordonnée, la phrase représente exactement le même agencement psychologique que si l'ordre de subordination était inverse, c'est-à-dire que si nous avions affaire à une subordination prédicative du type que nous avons étudié $[\ldots]:$ : quand j'avais treize ans, ma mère mourut ». (Séchehaye $1926: 202-203$ )

Dès le départ, l'idée d'un agencement psychologique reflétant l'ordre inverse de la «subordination » est donc invoquée.

Une dizaine d'années plus tard, Gougenheim (1938) emploie pour la première fois, selon Wilmet (2003 : 474), le terme de « subordination inverse $^{1} »$ appliqué au français. Cet auteur le définit ainsi :

On trouve des subordonnées commençant par que, après une proposition principale, qui, si l'on reconstruit la phrase avec une autre conjonction de subordination, deviennent principales tandis que la principale devient subordonnées [...] : Le cardinal n'avait pas gagné la porte, que ses larmes, violemment retenues, débordèrent (Chateaubriand, Mémoires d'Outre-Tombe, VI, 288) («Avant que le cardinal eût gagné la porte, ses larmes... débordèrent »). (Gougenheim $1938: 377$ )

Comme l'exprime bien cette citation, ce terme a été employé à l'origine pour caractériser certaines tournures introduites par que et il est justifié par une paraphrase «inversant» la «subordination ». Les deux citations précédentes se ressemblent fortement mais, dans cette dernière, G. Gougenheim ne fait pas le rapprochement avec les quand étudiés par A. Séchehaye. Cependant, ce rapprochement entre quand et que a été explicitement proposé par Sandfeld (1936) et Chétrit (1976).

Moins d'une trentaine d'années plus tard, un troisième terme (anglais celui-ci) a été proposé, selon Declerck (1997), par Labov\&Waletzky (1967) : il s'agit de «narrative clause ».Nous mentionnons ce terme car le rapprochement entre le «quand inverse » et le «quand narratif » est explicitement fait par Vogeleer (1998) et il met en lumière une propriété singulière des énoncés étudiés par A. Séchehaye et G. Gougenheim. Labov (1972 : 359-360) en donne la définitionsuivante :

We define narrative as one method of recapitulating past experience by matching a verbal sequence of clauses to the sequence of events which actually occurred.

Contrairement aux définitions précédentes, celle-ci met l'accent sur l'iconicité liée à l'ordre des constructions et non sur l'inversion du lien sous-jacent de «subordination». Cela se comprend, car la «subordination inverse », dans le cas où deux actions se succèdent, respecte généralement le principe d'iconicité. A l'origine, «subordination inverse » et « narrative clause » sont deux notions distinctes qui, par une subtile dérivation, tendront à se confondre. Néanmoins, la notion proposée par Labov (1972) a le mérite de ne pas introduire une contradiction interne en donnant la possibilité à une «subordonnée » de devenir la «principale».

Au niveau des critères, il n'est pas non plus évident d'y voir clair. En effet, les énoncés proposés par Sandström (1993 : 68-69) comportent des constructions en when antéposées alors que Declerck (1997) précise que les «narrative when-clauses » sont obligatoirement postposées. Ainsi, d'une même définition de départ (Labov 1972), ces deux auteurs abordent des constructions aux propriétés différentes.

De même, Vogeleer (1998) critique la commutation avec et alors (and then) proposée par Declerck (1997) en raison du fait que et alors est extérieur à l'intervalle associé à la situation précédente, ce qui n'est pas le cas, selon elle, du quand inverse. En fait, cette commutation semble appropriée mais elle ne s'applique pas aux constructions que Vogeleer (1998) nomme quand inverse. On mélange dans cette catégorie, au nom de

Même si nous savons que la « subordination inverse » est vraisemblablement issue du cum inversum latin. 
similitudes superficielles, tout ce que l'on n'a pas pu classer ailleurs. Du coup, on y trouve aussi bien des constructions qui représentent des actions se succédant sur le plan temporel (acceptant et alors) que des constructions en quand évoquant un événement qui s'insère à l'intérieur du cadre temporel posé précédemment (acceptant difficilement et alors), ce qu'illustrent les deux exemples suivants :

(5) Le petit garçon traversa des forêts d'eucalyptus, des landes de bruyères mauves, etc... Il dut se battre contre un sanglier... aperçut même une licorne. (Quand / Et alors) un matin, il arriva enfin au pied d'une très vieille montagne. (Vervin, cité par Eyot 1948)

(6) Jean lisait le journal (quand / ?et alors) Michel entra. (ex. Vogeleer 1998)

Le rapprochement entre quand et et (and) est d'ailleurs explicite dans la traduction suivante tirée des débats au parlement canadien :

(7) Le calme le plus complet régnait, quand tout à coup un agent de la GRC à qui on avait sans doute enjoint d'agir ainsi [...] s'est présenté et a procédé à l'arrestation du monsieur en question [...] / Thingswereverypeaceful, and all of a sudden an RCMP officerwhohad been told to do so [...] arrested the gentleman in question [...] (Hansard)

D'après ce que nous venons de voir, les définitions proposées ne s'appuient pas sur des analyses convergentes. Le dénominateur commun à tous ces termes, c'est une partie des exemples proposés en guise de représentants de la classe. Pour ce qui est des critères et des définitions, il existe des variations significatives illustrées notamment par le fait que les auteurs travaillant sur ce procédé «inverse » ne décrivent pas exactement les mêmes structures.

La notion de «subordination inverse » n'est donc pas employée de manière univoque, ce qui fait qu'il est difficile d'en cerner les contours avec exactitude. De plus, comme l'exprime bien la citation suivante, elle est parfois le résultat d'un mélange des niveaux d'analyse (visiblement entre syntaxe et sémantique) :

La proposition qui est formellement la principale présente en réalité le thème de la phrase et représente les circonstances (elle en indique le cas échéant le repérage temporel, par exemple la datation), c'est la subordonnée qui en est le propos et qui exprime le fait essentiel. (Riegel et alii $2002: 507$ )

Pour éviter ces deux écueils, nous préférons, dans les sections ci-dessous, décrire les exemples en distinguant les composantes sémantique et syntaxique et ne pas utiliser ce terme.

\section{Description sémantique}

Comme nous l'avons signalé dans l'introduction, il existe de nombreuses études portant sur l'analyse sémantique du quand présent dans les exemples tels que :

(8) Je pensais à nos deux vieux qui devaient dormir tranquillement, quand tout à coup j'entends souffler une machine sur la double voie. (conte)

On peut citer notamment Le Draoulec (2003) \& (2006), Vogeleer (1998), Borillo (1988), Maurel (1992), Baranzini (à paraître) (sur quando en italien), Declerck (1997) (sur when en anglais). En revanche, les études portant sur les exemples en avant que/de tels que :

(9) Lorsque, deux heures plus tard, Hadda s'est finalement présentée au tribunal, les policiers ont saisi la coupable affolée, lui ont arraché son chemisier en la maîtrisant, puis lui ont jeté un seau d'eau au visage. Avant de la traîner, à demi dévêtue, trempée et folle de trouille, dans tout le palais de justice. (presse)

sont plus limitées, l'analyse de cette seconde conjonction dans cette perspective étant relativement récente (cf. Le Draoulec [2003] \& [2006], Delort [à paraître ${ }_{a \& b}$, mais aussi Berrendonner et alii [1983])2 .

Dans ce qui suit, nous nous contentons de résumer les principaux résultats de ces études et travaillons uniquement sur les deux fonctionnements distincts illustrés par les exemples(1) à (4). Nous ne traitons pas des autres valeurs sémantiques qu'ils peuvent $\operatorname{avoir}^{3}$ et nous sommes conscients que la plupart des exemples sont ambigus et tolèrent les deux interprétations lorsque la «subordonnée » est postposée. Mais pour des raisons de clarté, nous limiterons systématiquement l'interprétation au sens le plus probable.

2 Nous renvoyons à ces travaux pour observer les argumentations précises.

3 Pour un panorama des autres valeurs de avant que/de, se référer à Le Draoulec (2001). 
Tout d'abord, précisons que les exemples contenant une localisation temporelle introduite par quand ou avant que n'articulent pas deux «propositions »(cf. Vogeleer, 1998 ; Le Draoulec, 2003).Dans ce cas, la « subordonnée » est assimilée à un constituant temporel. Et il est vrai que,dans les exemples suivants, les constructions et les constituants temporels de type prépositionnel qui les précèdent semblent se trouver dans la même position syntaxique et sont donc mutuellement substituables :

(10) En avril, quand on a lancé cette campagne sans précédent, $51 \%$ des boutiques vendaient des cigarettes à nos 'ados espions'. (presse)

(11) Alors ce que je voulais te dire dès hier avant que tu ne partes en trombe c'est que j'ai réussi à grappiller du budget afin de te proposer une prime (www)

En outre, il est clair que le contenu des deux «subordonnées » est présupposé ${ }^{4}$. Dans (10), le lancement d'une campagne sans précédent est présupposé et(11) présuppose que l'interlocuteur est parti en trombe. Afin de mettre en évidence cette propriété, on peut recourir à la loi d'enchaînement proposée par Ducrot (1991) qui dit que lorsqu'une situation est présupposée, il est impossible d'enchaîner directement sur celle-ci. Cela permet donc de distinguer l'exemple (12), dans lequel la situation exprimée par la construction en avant que est présupposée, de l'exemple $(13)^{5}$ :

(12) Je voulais te dire ça dès hier avant que tu ne partes en trombe. *Tu devais donc être très pressé.

(13) Après des heures d'âpres négociations, Loïc m'a donné son accord, avant que Paul vienne tout gâcher quelques minutes plus tard en lui révélant le subterfuge. J'aurais dû savoir que Paul ne pourrait pas tenir sa langue.

Mais quel est le statut sémantique de ce second fonctionnement?

Les constructions appartenant à cette catégorie ont une valeur assertive (cf. Le Draoulec 2003 \& 2006). La succession temporelle s'opère sur le même plan et il y a une progression de la narration. On peut du reste les paraphraser par (et) ensuite. En voici encore quelques exemples :

(14) Le porte-parole a commencé par se réjouir au nom du gouvernement avant de se raviser et signifier s'être exprimé en son nom personnel. Puis, dans la soirée, le gouvernement s'est finalement félicité. (Libération, cité par Le Draoulec 2006)

(15) Au début cela aurait dû être la chronique heureuse d'un tournage, la matière d'un bonus sur le futur DVD d'un grand film à succès... Au début, seulement... avant que tout ne disparaisse, avant que les sept plaies d'Egypte ne s'abattent sur Terry Gilliam et sur son rêve le plus démesurément fou. Alors cette caméra innocente qui aurait dû, dans l'idéal, n'être là que pour les instants de grâce et de joie, devint le témoin impuissant d'une débâcle. (Journal du cinéma Utopia, 3-30 septembre 2003, cité par Le Draoulec2003)

Dans ce dernier exemple, l'énoncé qui commence par alors enchaîne d'ailleurs sur le contenu des constructions en avant que, ce qui montre bien son caractère non présupposé.

En ce qui concerne les Quand-C, il existe une similitude saisissante au niveau du contraste entre présupposition et assertion. En effet, on peut exclure la valeur présuppositionnelle, dans des exemples tels que (16), en signalant que le caractère inattendu va à l'encontre de la notion de connaissance préalable, même si cela ne constitue pas une condition nécessaire et suffisante. En guise d'indice du caractère non présupposé, on peut sans difficulté enchaîner directement avec un énoncé exprimant une conséquence de la situation exprimée dans la «subordonnée ». Il est par exemple possible de faire directement suivre :

(16) Ils se préparaient à gagner l'intérieur de l'île en remontant le rio, quand, soudain, une ombre se dressa devant eux. (roman)

de l'énoncé «Ils dégainèrent aussitôt leurs épées ».

Les Quand-C de ce type sont également assertives et disposent donc d'une autonomie énonciative. Le temps de référence est courant et appartient à « l'avant-plan de la narration » (Declerck, 1997) alors que le temps de référence exprimé dans les «subordonnées temporelles canoniques » est subsidiaire (Le Draoulec 1997). Quand a alors un rôle de « connecteur » établissant une relation temporelle entre deux propositions assertées.

$4 \quad$ Pour une discussion sur les acceptions de présupposition et d'assertion, qui ne sont pas univoquement définies, voir les travaux d'A. Le Draoulec cités dans la bibliographie.

5 Dans cet exemple, la présence de quelques minutes plus tard marque en plus une avancée de la narration, ce que l'on ne pourrait pas avoir si la situation était présupposée. 
Il existe tout de même des petits effets sémantiques supplémentaires que ne connaît pas avant que/de. L'action exprimée dans la «subordonnée » a un caractère inattendu et surprenant. Elle vient interrompre l'action décrite dans la «principale » avec souvent un effet de surprise. De plus, il a été noté par Vogeleer (1998) que cette interprétation est liée à la possibilité d'attribuer un point de vue perceptuel au personnage représenté dans la « principale ».

Pour finir, on peut signaler qu'il existe une dimension iconique (cf. citation de Labov [1972] ci-dessus), à savoir que l'ordre des événements correspond à l'ordre des constructions verbales, la « subordonnée » devant se situer obligatoirement après la «principale » compte tenu du fait qu'elle contient un événement ultérieur.

Cependant, contrairement à l'analyse sémantique, que nous venons de détailler, la caractérisation syntaxique de la relation liant la « subordonnée » à la «principale » reste, à notre avis, relativement méconnue. Le Draoulec (2003) parle d' " une relation de succession temporelle [...] aussi proche de la connexion (entre deux propositions syntaxiquement indépendantes) que de la subordination », sans que l'on sache réellement quelle est la nature de ce lien.

Le problème principal repose sur la polysémie du terme «subordination » mis en lumière dans la citation suivante :

Anscombre et Ducrot ont aussi montré qu'il existe deux mais en français, le mais opérateur (correspondant à sino et sondern), lié à une "subordination sémantique », et le mais connecteur (correspondant à pero et aber), lié à une « coordination sémantique » (Simon \&Grobet 2002)

ou dans le titre de l'article de Culicover\&Jackendoff (1997) : semantic subordination despitesyntactic coordination.

Nous pensons qu'employer le même terme pour désigner tantôt une relation sémantique, tantôt une relation syntaxique ne peut que nuire à la clarté de l'argumentation. De plus, la reconnaissance de la «subordination » dans son acception syntaxique se limite encore trop souvent à la présence de avant que ou quand, comme l'exprime bien la citation suivante :

Ce n'est donc plus la subordonnée qui éclaire du point de vue du temps le verbe principal, mais plutôt l'élément antéposé qui précise le procès régi, qui reste malgré tout subordonné puisqu'il est introduit par quand (ou lorsque). ${ }^{6}$ (Chétrit $\left.1976: 110\right)$

Nous allons maintenant montrer que cette conception «morphologisante » est trop restrictive et empêche de mettre en évidence les différences de fonctionnements syntaxiques entre les deux types étudiés ici, qui pourtant sont manifestes.

\section{Description syntaxique}

Pour les deux raisons exposées ci-dessus (polysémie et critère de reconnaissance), nous éviterons dans ce qui suit d'employer le terme «subordination ». Nous utiliserons à sa place le terme de « rection »au sens de relation qui lie deux éléments dont l'un (le recteur) exerce une contrainte grammaticale sur l'autre (le régi). Notre conception de la rection, inspirée de l'approche pronominale (Blanche-Benveniste et alii 1987), est donc plus large que l'emploi «standard » de ce terme et permet d'englober tous les éléments qui sont dépendants d'un verbe. Ainsi, certains «circonstanciels » de même que les compléments faisant partie de la valence (i.e. sélectionnés par le verbe) sont considérés comme régis ${ }^{7}$.

Mais avant de commencer l'analyse syntaxique proprement dite, nous souhaitons mettre en lumière un phénomène intéressant, à savoir la présence d'une ponctuation forte entre les deux constructions verbales, constatée tout particulièrement dans les copies d'élèves de primaire.

(17) Tous les deux partirent dabord regarder à côté des maisons, ensuite dans la forêt. Quand à ce moment ils entendirent du bruit !!! C'était CorninBruchon qui courait après une jeune fille. (Enfant de 10 ans, cité par Sabio2003)

Le premier à avoir relevé ce fait est Eyot (1948) et il considérait comme fautive cette utilisation apparemment singulière de la ponctuation.

$6 \quad$ C'est nous qui soulignons.

$7 \quad$ Les tests utilisés pour mettre en évidence la rection sont détaillés plus loin. 
Toute l'année, j'ai corrigé dans les rédactions de mes élèves de quatrième et de cinquième des propositions indépendantes introduites par quand.

Quelques décennies plus tard, c'est Berrendonner (1990) puis Sabio (2003) qui signalent eux aussi cette tournure dans des copies d'élèves. Ce dernier auteur associe ce tour à «l'écriture cérémonieuse » et il précise que les détachements graphiques observés n’ont rien de «fantaisistes mais illustrent de manière éclairante [leur] statut grammatical particulier ». A cinquante-cinq ans d'intervalle, le regard porté sur ces tournures a donc fortement changé, passant d'un usage fautif à un procédé accompagnant le statut grammatical de ces constructions. Le Draoulec (2006) en fait même un indice de reconnaissance. Les élèves ayant produit ces énoncés ont donc sans doute eu l'intuition saisissante d'une autonomie plus grande que les Quand-C temporelles canoniques.

En outre, la présence de la ponctuation forte se vérifie aussi pour les exemples en avant que/de:

(18) Les fils et filles de ceux qui avaient demandé et obtenu la départementalisation en 1946, dans la foulée de l'aprèsguerre se sont sentis ensuite «floués ». Et se sont battus pour l'autonomie (années 70), puis pour la décentralisation (années 80) et pour l'égalité (années 90). Avant d'entamer maintenant un nouveau combat pour «le développement » et la prise en compte des réalités régionales au sein de l'Union européenne. (presse)

On comprend cependant le désarroi de cet enseignant confronté à des « indépendantes » introduites par un « subordonnant » qu'il analysait en ces termes :

Il garde manifestement une valeur de liaison, qui me semble plus forte qu'une simple coordination, à mi-chemin de la subordination, à peu près équivalente de celle du relatif de liaison latin - ou français. (Eyot 1948)

J. Pohl, dans le même numéro de la revue Le Français Moderne, a une conception bien différente. Il suggère, dans sa réponse reproduite à la fin de l'article d'Antoine (1948 : 274), que la construction en quand n'est ni subordonnée, ni coordonnée mais indépendante. Il avance donc avec prudence que quand est employé avec une «rupture de subordination». De même, Sandfeld (1936:263) dit de ces constructions qu'elles se « détachent » et deviennent « autonomes ».

Si l'on accepte, comme nous le proposons, de faire reposer la détermination de la rection sur d'autres critères que la présence de la conjonction, les conclusions que l'on en tire vont effectivement dans le sens d'une autonomie syntaxique. Les tests que nous utilisons pour mettre en évidence le lien de rection sont ceux que l'on trouve dans de nombreux cadres syntaxiques descriptifs (dans Blanche-Benveniste et alii [1987] et Creissels [2006] par exemple). Nous en avons principalement trois : l'équivalence avec une proforme (ici le quand interrogatif), l'extraction entre c'est et que et le contraste (ou la portée) des modalités. Afin d'illustrer l'utilisation de ces trois tests, nous proposons l'exemple suivant qui les présente dans l'ordre :

(19) Quand j'étais adolescente, David Hamilton était très à la mode. (roman)

a. Quand David Hamilton était-il très à la mode ? Quand j'étais adolescente.

b. C'est quand j'étais adolescente que David Hamilton était très à la mode.

c. David Hamilton était très à la mode, mais pas quand j’étais adolescente.

Etant donné que les trois énoncés ci-dessus sont grammaticaux, nous dirons que la Quand-C est régie. Mais il n'en va pas de même pour toutes les Quand-C. Voici le résultat des tests lorsqu'on les applique sur notre exemple (1), repris ici :

(20) Pécuchet venait d'en remettre la note à Bouvard quand tout à coup le tonnerre retentit et la pluie tomba (roman)

a. Quand Pécuchet venait-il d'en remettre la note à Bouvard ? ${ }^{*}$ Quand tout à coup le tonnerre retentit et la pluie tomba.

b. ?C'est quand tout à coup le tonnerre retentit et la pluie tomba que Pécuchet venait d'en remettre la note à Bouvard.

c. ? Pécuchet venait d'en remettre la note à Bouvard, mais pas quand tout à coup le tonnerre retentit et la pluie tomba.

Dans ce cas-là, nous dirons que la Quand-C n'est pas régie.

En complément de ces indices, on peut ajouter la possibilité pour la seconde construction d'être dotée de sa modalité interrogative propre :

J'étais en train de m'impatienter, quand, tout à coup, qu'est-ce que j'entends ? (Leroux, cité par Sandfeld 1936 : 264) 
et l'impossibilité, au niveau de l'ordre linéaire, d'apparaître en position initiale ${ }^{8}$ :

(22) ? Quand tout à coup le tonnerre retentit et la pluie tomba, Pécuchet venait d'en remettre la note à Bouvard.

De plus, les contraintes internes qu'impose normalement quand cessent d'opérer, ce qui dans la littérature anglo-saxonne, a reçu le nom de «main clause phenomena ». Par exemple, la Quand-C peut ne pas être une construction verbale :

Je cherche entre les voitures, au cas où un de ces sales gosses que je croise de temps en temps se serait caché quand soudain... PLOC... un bruit sourd dans mon dos. (www)

L'analyse en termes d'autonomie morphosyntaxique de ces unités n'est absolument pas révolutionnaire. D'autres chercheurs ont déjà montré, avant nous, que toutes les constructions introduites par des « conjonctions de subordination » ne sont pas nécessairement « subordonnées »:

As we have already indicated by calling them clause combinations, we do not think that our examples can be interpreted as clauses embedded within other clauses. In other words, these clauses do not function as adverbials (or adjuncts). (Matthiessen\& Thompson 1988)

Et la même analyse a été proposée pour when en anglais :

The when clause is not a subordinate clause but a main clause and when can be treated as a conjunction joining two main clauses.(Miller \&Weinert 1998)

Il est ainsi possible de montrer que la distinction sémantique proposée pour les deux constructions ci-dessus va de pair avec une distinction syntaxique fondamentale : l'unité introduite par quand est régie dans (19) et non régie dans (20).

Il semblerait donc que l'autonomie énonciative relevée par Le Draoulec (2003) soit congruente avec une autonomie syntaxique. Et on observe la même distinction pour les exemples en avant que/de, signe qu'il ne s'agit pas d'un fonctionnement propre à quand.

En effet, les constructions en avant que/de contenant un événement présupposé sont clairement régies :

(24) Je ferai mes devoirs avant que la nuit tombe.

a. Quand feras-tu tes devoirs ? Avant que la nuit tombe.

b. C'est avant que la nuit tombe que je ferai mes devoirs.

c. Je ferai mes devoirs, mais pas avant que la nuit tombe.

On en trouve même directement en position de complément de verbe :

(25) Si ça s'est passé avant que tu sois avec ton copain actuel, ça ne le regarde pas ! (www)

En revanche, les constructions assertives ne sont pas régies :

(26) Les recettes de privatisation ont atteint leur maximum en 1991, avant de décliner fortement, reflétant le fait que les grands pays latino-américains se sont désengagés du secteur public dès le début de la décennie. (presse)

a. Quand les recettes de privatisation ont-elles atteint leur maximum en 1991 ? *Avant de décliner fortement, reflétant le fait que les grands pays latino-américains se sont désengagés du secteur public dès le début de la décennie.

b. ?C'est avant de décliner fortement, reflétant le fait que les grands pays latino-américains se sont désengagés du secteur public dès le début de la décennie que les recettes de privatisation ont atteint leur maximum en 1991.

c. ? Les recettes de privatisation ont atteint leur maximum en 1991, mais pas avant de décliner fortement, reflétant le fait que les grands pays latino-américains se sont désengagés du secteur public dès le début de la décennie.

Et elles aussi apparaissent difficilement en position initiale :

(27) ? Avant de décliner fortement, reflétant le fait que les grands pays latino-américains se sont désengagés du secteur public dès le début de la décennie, les recettes de privatisation ont atteint leur maximum en 1991.

8 Cela est parfois possible mais le sens ainsi que l'analyse syntaxique sont alors différents. 
Pour appuyer cette distinction, on peut recourir à un éclairage diachronique. En effet, la polyfonctionnalité des conjonctions en français contemporain est parfois le résultat de deux usages anciennement distincts qui sont venus se confondre. C'est ce qui semble être le cas avec avant que/de, si l'on se réfère aux exemples de moyen français proposés par Combettes (2006: 103) :

\begin{abstract}
un petit avant ce quemesires Robert d'Artois et la contesse de Montfort se departesissentdouhavene de Plumude, uns granstourmens se mist sus mer, (...) et furent plus de quinze jours waucrant sus la mer (...) avant que il se peuissent tout remetreensamble (Froissart, 1400) / peu avant que monseigneur ... et ... partent du port de Plumude, une grande tempête se leva sur la mer ... et ils furent plus de quinze jours errant sur la mer avant qu'ils puissent se retrouver
\end{abstract}

L'auteur en propose l'analyse suivante :

La première locution, qui contient le démonstratif, introduit une subordonnée qui fonctionne comme élément thématique en début d'énoncé et constitue un cadre de discours, apportant une information qui demeure dans le domaine strictement chronologique, n'ajoutant pas d'indications qui pourraient renvoyer à une liaison logique des événements, par exemple ; la subordonnée qui suit avant que, en revanche, marque davantage la liaison qui est établie entre les contenus des propositions, soulignant, en l'occurrence, la durée nécessaire (plus de quinze jours) à la réalisation d'un procès (pouvoir se retrouver). (Combettes 2006 : 103-104)

L'un des deux fonctionnements que l'on retrouve en français contemporain viendrait donc de avant ce que alors que l'autre serait issu de avant que.

Cette distinction syntaxique fondamentale entre dépendance et autonomie grammaticalesétant posée, il nous reste encore à exposer nos perspectives et l'éventail des fonctionnements dont il nous faudra rendre compte. Ces emplois nous permettront, lorsd'une prochaine étude, de vérifier de manière étendue les liens qu'il existe entre les caractérisations syntaxiques et sémantiques.

\title{
4. Conclusion \& perspectives
}

Pour l'heure, il est impossible de dire s'il existe une corrélation systématique entre présupposition et rection et entre assertion et indépendance grammaticale. Sans trop nous avancer, nous pouvons néanmoins dire que les assertions faisant progresser la narration sont toujours non régies. Mais pour les autres cas, les subtilités sémantiques auxquelles il faut faire face, avec avant que/de notamment, rend l'analyse extrêmement complexe. Nous listons dans ce qui suit les cas qu'il nous faudra traiter.

Tout d'abord, on peut citer les constructions en avant que/de présentant un événement que Le Draoulec (2001) qualifie de présupposition «contrefactuelle » par opposition aux présuppositions «factuelles » vues cidessus. Cette interprétation est présente dans l'exemple suivant, emprunté à Berrendonner et alii (1983).

$$
\text { Tous les défenseurs moururent avant de se rendre (Le Monde) }
$$

Néanmoins, avant de étant polysémique, le journal Le Canard Enchaîné, friand de ces ambiguïtés de langage, enchaîna ainsi :

$$
\text { et ils furent ensuite massacrés avant d'être emprisonnés (Canard Enchaîné) }
$$

Ces deux exemples tolèrent bien évidemment deux interprétations dont l'une est plausible et l'autre quelque peu invraisemblable. Avec le sens «contrefactuel», «les défenseurs n'ont pas eu le temps de se rendre », alors qu'avec l'autre acception, «ils se sont rendus après leur mort ». Il suffit de rajouter un verbe marquant une modalité accomplie (avoir pu), pour faire disparaître complètement la seconde interprétation :

Tous les défenseurs moururent avant d'avoir pu se rendre.

Il nous faudra donc voir si, sur le plan syntaxique, il existe des propriétés permettant de distinguer les événements factuels des contrefactuels ou s'il s'agit d'un phénomène purement sémantique.

$\mathrm{Au}$ niveau des autres valeurs sémantiques que l'on rencontre avec avant que/de, il en existe une sémantiquement proche de sinon:

$$
\text { Tu me passes le sel avant que je te casse la tête. }
$$

et une autre sémantiquement proche de pour que : 

russes! (www)

Il existe aussi des constructions en avant que et en quand portant sur l'énonciation et non sur l'énoncé. En termes positionnels, il est intéressant de noter que l'une est bloquée en position initiale :

(34) Ah oui, avant que j'oublie, j'ai des propositions de fausses pub à mettre sur le site officiel. (www)

a. ? J'ai des propositions de fausses pub à mettre sur le site officiel, avant que j'oublie.

alors que l'autre est apparemment mobile :

(35) Quand on y pense ... mieux vaut ne pas trop se poser de questions (www)

a. Mieux vaut ne pas trop se poser de questions, quand on y pense

Face à cette profusion de valeurs sémantiques, il faudra aussi tenter de répondre à la question de l'unicité de avant que/de et quand à travers le spectre de la polysémie ou de l'homonymie de ces morphèmes.

Pour conclure, nous pouvons affirmer que, sur le plan de la syntaxe, de nombreuses conjonctions possèdent les deux fonctionnements - régis et non régis (cf. Debaisieux 2006) - ce qui confère à notre analyse une justification externe et une certaine généralité. Mais comme nous l'avons montré dans cette dernière section, desinvestigations supplémentairesdoivent être menéesafin de pouvoir analyser syntaxiquement l'ensemble des valeurs prises par avant que.

\section{Références}

Baranzini, L. (à paraître). Italien quando : un cas de subordination inverse, Cahiers Chronos.

Benzitoun, C. (2006). Examen de la notion de subordination. Le cas des quand "insubordonnés », in: G.Rebuschi ; I. Bril, (dir.), Coordination et subordination : typologie et modélisation, Faits de Langues 28 : $35-46$.

Berrendonner, A. (1990). Pour une macro-syntaxe, in: D. Willems (éd.), Données orales et théories linguistiques, Travaux de linguistique $21: 25-31$.

Berrendonner, A.; Le Guern, M.; Puech, G. (1983).Principes de grammaire polylectale, Lyon : Presses Universitaires de Lyon.

Blanche-Benveniste, Cl. ;Deulofeu, J. ;Stéfanini, J. ; Eynde, K. van den (1987).Pronom et syntaxe. L'approche pronominale et son application à la langue française, Paris : SELAF.

Borillo, A. (1988).Quelques remarques sur quand connecteur temporel, Langue Française 77 : 71-91.

Chétrit, J. (1976).Syntaxe de la phrase complexe à subordonnée temporelle, Paris : Klincksieck.

Creissels, D. (2006).Syntaxe générale, une introduction typologique, Tome 2 la phrase, Hermès Lavoisier.

Combettes, B. (2006). La grammaticalisation des locutions conjonctives : l'opposition que/ce que, in: G.Rebuschi ; I. Bril (dir.), Coordination et subordination : typologie et modélisation, Faits de Langues28 : 95-105.

Culicover, P. W. ;Jackendoff, R. S. (1997).Semantic subordination despitesyntactic coordination, LinguisticInquiry 28/2 : 195-218.

Debaisieux, J.-M. (2006). La distinction entre dépendance grammaticale et dépendance macrosyntaxique comme moyen de résoudre les paradoxes de la subordination, in: G.Rebuschi; I. Bril (dir.), Coordination et subordination : typologie et modélisation, Faits de Langues 28 : 119-132.

Declerck, R. (1997). When-Clauses and Temporal Structure, London :Routledge.

Delort, L. (à paraître ${ }_{\mathrm{a}}$ ).Etude du rôle de avant que dans la structure du discours, Cahiers Chronos.

Delort, L. (à paraître ${ }_{\mathrm{b}}$ ). Exploring the Role of Clause Subordination in Discourse Structure - The Case of French Avant que, in : C. Fabricius-Hansen; W. Ramm (eds), Subordination vs. Coordination in Sentence and Text, Volume of Studies in Language Companion Series, Amsterdam/Philadelphia : John Benjamins.

Ducrot, O. (1991).Dire et ne pas dire, Paris : Hermann.

Eyot, Y. (1948).Quand..., Le français moderne 16 : 108.

Gougenheim, G. (1938).Le système grammatical de la langue française, Paris : Bibliothèque du français moderne.

Labov, W. (1972). The transformation of experience in narrative syntax, in :W.Labov (ed.), Language in the Inner City, Philadelphia: University of Pennsylvania Press, 354-396. 
Labov, W. ;Waletzky, J. (1967). Narrative analysis : oral versions of personal experience, in : J. Helm (ed.), Essays on the Verbal and Visual Arts, Proceedings of the 1966 Annual Spring Meeting of the American Ethnological Society, Seattle: University of Washington Press, 12-44.

Le Draoulec, A. (2006). De la subordination à la connexion temporelle, in : E. Moline ; D.Stosic ; C.Vetters (textes réunis par), Les connecteurs temporels du français, Cahiers Chronos 15, Amsterdam - New York : Rodopi, 39-62.

LeDraoulec, A. (2003).Quand, jusqu'à ce que et avant que : quelques cas particuliers de subordination temporelle hors présupposition, in : E. Comès; E. Hrubaru (eds), Dix ans de Séminaire de Didactique Universitaire - Recueil anniversaire, Université Ovidius Constanta : EdituraUniversitaria Craiova, 175196.

LeDraoulec, A. (2001). Les différents types d'interprétation des subordonnées introduites par avant que,in : R. Bok-Bennema ; B. de Jonge ; B. Kampers-Manhe ; A. Molendijk (eds), Adverbial Modification, 157-168.

LeDraoulec, A. (1997). Etude présuppositionnelle des subordonnées temporelles, Thèse de sciences du langage, Université de Toulouse - Le Mirail.

Matthiessen, C. ; Thompson, S. (1988). The structure of discourse and 'subordination', in :J.Haiman ; S. Thompson (eds), Clause combining in grammar and discourse, Typological studies in language 18, Amsterdam/Philadelphia: John Benjamins, 275-329.

Maurel, J.-P. (1992). Subordination inverse et neutralisation du relatif, in : J. Chuquet; D.Roulland (dir.), Subordination, Travaux linguistiques du Cerlico5, Presses Universitaires de Rennes, 72-88.

Miller, J. ;Weinert, R. (1998). Spontaneous spoken language. Syntax and discourse, Oxford : Clarendon Press.

Pohl, J. (1948). Réponse citée dans Antoine (1948), Le français moderne $16: 274$.

Rebuschi, G. ; Bril, I. (dir.), (2006). Coordination et subordination : typologie et modélisation, Faits de Langues 28, Ophrys.

Riegel, M. ;Pellat, J.-C. ;Rioul, R. (2002).Grammaire méthodique du français, Coll. Linguistique nouvelle, Presses Universitaires de France.

Sabio, F. (2003). L'écriture cérémonieuse chez les enfants : quelques exemples d'intégration grammaticale, in : E.Ferreiro ; M. Pascucci (éds), Rivista di psicolinguisticaapplicata, special issue, 79-90.

Sandfeld, K. (1936).Syntaxe du français contemporain, Tome II : Les propositions subordonnées, CopenhagueParis : Librairie E. Droz.

Sandström, G. (1993). When-clauses and the temporal interpretation of narrative discourse, University of Umeå, Report 34.

Séchehaye, A. (1950).Essai sur la structure logique de la phrase, Paris : Champion.

Simon, A.-C. ; Grobet, A. (2002). Intégration ou autonomisation prosodique des connecteurs, Speech Prosody 2002, Aix-en-Provence.

Vogeleer, S. (1998).Quand inverse, Revue Québécoise de linguistique 26/1 : 79-101.

Wilmet, M. (2003).Grammaire critique du français, Duculot : Bruxelles. 